doi:10.1017/S0022215116005387

\section{ID: IP042}

"Phone a friend" - three challenging cases, one invaluable friend

Presenting Author: Sherif Habashi

Sherif Habashi, Daniel Gjoni, Nazan Can Guru Naidu

Royal Free London NHS Foundation Trust

Learning Objectives: Developing a close relationship with a senior colleague who is readily accessible in an emergency early in one's career can pay huge dividends.

Introduction: No matter how long a surgeon has been in practice, from time to time they will inevitably find themselves in an unexpected situation where they are in difficulty and out of their depth. At such times it is vital to know where to turn for help.

Method: We describe three cases over a 20 year period which illustrate the value of having such a friend and colleague to turn to.

Case 1: a 60 year old female who had undergone a modified radical mastoidectomy over 20 years previously. She had had several episodes of facial palsy associated with an infected mastoid cavity which had resolved after microsuction under GA. On this occasion a complete palsy persisted for 3 weeks despite microsuction and a decision was made to explore the ear. The nerve was found to be dehiscent and attenuated to less than a quarter of its normal calibre at the second genu. Telephone advice was to excise the damaged segment, mobilise, reroute and perform primary end to end anastomosis.

Case 2: a 50 year old female presented with a persistent ear discharge after grommet insertion for a middle ear effusion. CT imaging suggested chronic mastoiditis, however, upon exploration a dehiscent tegmen and middle fossa dura was found with herniated temporal lobe and profuse CSF leakage. Telephone advice was to resect the herniated brain, repair the dura with fascia, crushed muscle and Surgicel before obliterating the mastoid.

Case 3: a 10 year old girl undergoing tympanoplasty had life-threatening bleeding from a dehiscent and massively dilated sigmoid sinus. Telephone advice was to repair the leak with fascia, crushed muscle, Floseal, Surgicel and bone wax.

Results: The first patient recovered facial nerve function to HB grade 2-3. The second and third patients had uneventful and complete recoveries without the need for further surgery.

Conclusion: Contacting an experienced colleague by telephone from the operating theatre can often save the day.

doi:10.1017/S0022215116005399

\section{ID: IP043}

Ten years after the $7 / 7$ bombings - an otologic perspective
Presenting Author: Sherif Habashi

Sherif Habashi, Ghous Handoo

Royal Free London NHS Foundation Trust

Learning Objectives: Physical injuries suffered by victims of terrorism may be identical to those caused by accident. However, the psychological Impact of terrorism may worsen the prognosis for fully recovery. It is important that victims of terrorism receive the most expert assistance at the earliest possible stage if they are to have the best chance of complete recovery from their physical injuries.

Introduction: The London bombings of 7th July 2005 left 56 dead (including the perpetrators) and over 700 injured. Ear damage is extremely common following exposure to explosions. Those injuries include tympanic membrane rupture, which may or may not heal spontaneously, sensorineural hearing loss, tinnitus, and vestibulopathy. The prognosis is variable and may be influenced by many factors.

Method: We present three patients treated in our institution who each suffered ear damage during the $7 / 7$ bombings.

Case 1: a 19 year old autistic man sustained a ruptured left tympanic membrane and when initially seen had an actively discharging middle ear. Microsuction was performed and topical antibiotic drops prescribed.

Case 2: a 60 year old civil servant sustained a central tympanic membrane perforation with associated mixed hearing loss and tinnitus. After this failed to heal tympanoplasty was performed.

Case 3: a 21 year old lady sustained bilateral tympanic membrane perforations which failed to heal. She underwent surgery on both ears including revision on the first side.

Results: The first patient's tympanic membrane healed spontaneously but he represented several times with otitis externa in the previously damaged ear. The second patient's surgery was successful in closing the perforation but he is left with persistent tinnitus and is now using hearing aid. The third patient had and iatrogenic middle ear cholesteatoma following her first operation resulting in ossicular erosion. After revision surgery and repair of the contra lateral perforation she had been left with mild bilateral hearing loss. 10 years on she has tinnitus which at times is disabling, recurrent BPPV and chronic imbalance.

Conclusion: Blast injury to the ear can result in a spectrum of injuries which may or may not leave lasting disability. The psychological trauma inflicted on victims of terrorism can have a significant impact on their ability to cope with tinnitus and vestibular symptoms.

doi:10.1017/S0022215116005405

\section{ID: IP044}

Life Threatening Bleeding during

Tympanoplasty in a Child

Presenting Author: Sherif Habashi 